Check for updates

Cite this: RSC Adv., 2017, 7, 18231

Received 17th February 2017

Accepted 13th March 2017

DOI: $10.1039 / c 7 r a 02002 c$

rsc.li/rsc-advances

\section{Factors affecting $p$-nitrophenol removal by microscale zero-valent iron coupling with weak magnetic field (WMF) $\uparrow$}

\author{
Juanshan Du, Di Che, Xiaofan Li, Wanqian Guo (D) and Nanqi Ren*
}

The effect of WMF on the kinetics of $p$-nitrophenol (PNP) removal by six commercial zero-valent iron (ZVI) samples from different origins were studied at $\mathrm{pH}$ 4.0. The pseudo-first-order rate constant $\left(k_{\text {obs }}\right)$ of PNP removal by ZVI with WMF were 2.9-5.4-fold greater than those without WMF. The strong correlation between the specific reaction rate constants $\left(k_{S A}\right)$ of PNP removal by various ZVI samples and the specific rate constant of $\mathrm{Fe}(I)$ release ( $k_{\mathrm{Fe}}(\mathrm{II})$ release $\left.\mathrm{SA}\right)$ during these processes indicated that enhancement of PNP removal by ZVI in the presence of WMF was mainly ascribed to the improved $\mathrm{Fe}^{0}$ corrosion and Fe(II) generation. Effects of $\mathrm{pH}$ value (4.0-7.0), ZVI loading (100-1000 $\mathrm{mg} \mathrm{L}^{-1}$ ), PNP concentration (5$100 \mu \mathrm{M})$, magnetization time (1-120 min), and various anions (at 1-50 $\mu \mathrm{M}$ concentration) on PNP removal by $Z \mathrm{VI}$ with and without the presence of WMF was investigated. The presence of WMF significantly accelerated PNP removal at $\mathrm{pH} 4.0-7.0$, especially at neutral $\mathrm{pH}$ values. The $k_{\mathrm{SA}}$ and

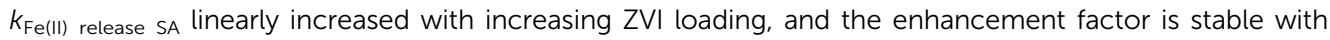
increasing ZVI loading. PNP concentration exhibited a very slight effect on PNP removal with and without WMF. The $k_{\text {obs }}$ of PNP removal increased with increased magnetization time and trended to be consistent for more than 5 min of magnetization by WMF. WMF exhibited a positive effect on PNP removal in the presence of sulfate, chlorate, and nitrate. Although perchlorate could inhibit PNP removal by ZVI, WMF decreased the negative effect of perchlorate on PNP removal by ZVI. Furthermore, the possible degradation pathway of PNP degradation by ZVI was proposed according to the detected intermediates.

\section{Introduction}

The increasing occurrence of organic chemical pollution in the aquatic environment, reported by various studies, has also raised widespread concern over its adverse effects on aquatic ecology and risks to human health. ${ }^{1}$ Nitrophenols, an important category of industrial raw materials, have been widely used as precursors or intermediates in the production of pesticides, pharmaceuticals, synthetic dyes, explosives, and rubber chemicals. $^{2-4}$ Three nitrophenols (2-nitrophenol, 4-nitrophenol, and 2,4-dinitrophenol) have been listed as priority pollutants in water by the U.S. Environmental Protection Agency (EPA) since 1979 for their toxicity, biorefractory effects, potential carcinogenicity, and mutagenicity. ${ }^{5}$ Usually, nitrophenols have possessed strong resistance to chemical and biological oxidation for the electron-withdrawing nitro group on the benzene ring; nevertheless, nitrophenols are reduced to limited products

State Key Laboratory of Urban Water Resource and Environment, Harbin Institute of Technology, Harbin 150090, PR China. E-mail: guowanqian@126.com; rnq@hit. edu.cn; Fax: +86-451-86283008; Tel: +86-451-86283008

$\dagger$ Electronic supplementary information (ESI) available. See DOI: $10.1039 / \mathrm{c} 7 \mathrm{ra02002c}$ of nitrosophenols or aminophenols by anaerobic biological treatments, which still exhibit environmental risks. ${ }^{6}$ As a priority nitrophenol, 4-nitrophenol (p-nitrophenol or PNP) is easily released to water ${ }^{7}$ and soil ${ }^{2}$ during its production and application due to its high stability and solubility, and it has received wide attention for its hazardous effects on the blood, liver and central nervous system of human beings. ${ }^{8}$ Thereby, it is essential to control the concentration of PNP in aquatic environments by developing efficient technologies.

Many studies have employed that various techniques, such as advance oxidation process (AOPs), ${ }^{9}$ adsorption, ${ }^{10}$ and reduction, ${ }^{11}$ to control PNP in water and wastewater treatment processes. Zero-valent iron ( $\mathrm{ZVI}$ or $\left.\mathrm{Fe}^{0}\right)$, as a green reductive reagent, has shown the combined effects of reduction, adsorption, co-precipitation and oxidation on contaminant degradation in water. ZVI has been widely applied to remove various pollutants such as azo dye, nitroaromatics, halogenated organic compounds, nitrate, perchlorate, and many heavy metals since 1996. ${ }^{12-15}$ Generally, the contaminant degradative rate of ZVI is dependent on the surface area and abundant reactive surface sites of ZVI samples. Thus, nanoscale ZVI (nZVI) has been proposed to replace the microscale ZVI to improve the performance of $\mathrm{Fe}^{0} .^{16}$ However, the synthesis of nZVI has been 
considered too expensive for the costly reagents and complex processes. ${ }^{17}$ Besides, nZVI surface passivation should be carried out before handling due to its high reactivity, and the potential toxicity of nZVI and derivatives has raised much concern. ${ }^{18}$ Recently, Guan et al. ${ }^{\mathbf{1 9 , 2 0}}$ have done many comprehensive studies on weak magnetic field (WMF) enhancing the removal of several heavy metals by microscale ZVI. The application of an inhomogeneous weak magnetic field (WMF) $\left(B_{\max }<20 \mathrm{mT}\right)$ could significantly enhance Se(Iv) removal by both pristine ZVI and aged $\mathrm{ZVI}^{19,20}$ and greatly improve $\mathrm{As}(\mathrm{v})$ and $\mathrm{As}(\mathrm{III})$ removal by $\mathrm{Fe}^{0}$ at $\mathrm{pH}_{\mathrm{ini}} 3.0-9.0 .^{21}$ The accelerated $\mathrm{Se}(\mathrm{IV}), \mathrm{As}(\mathrm{III})$ and $\mathrm{As}(\mathrm{v})$ removal by $\mathrm{Fe}^{0}$ was mainly ascribed to the improved $\mathrm{Fe}^{0}$ corrosion and $\mathrm{Fe}(\mathrm{II})$ generation. Besides, premagnetized multiple ZVI samples also enhanced the degradative rates of various heavy metals, and the strong correlations between the $\mathrm{Fe}(\mathrm{II})$ generation rates of various pristine $\mathrm{ZVI} /$ premagnetized ZVI samples and the removal rate constants of a specific contaminant by these ZVI samples was also observed. ${ }^{22}$ Although very few studies have been conducted to investigate heavy metals removal by microscale ZVI in the presence of WMF, the limited information revealed the efficient removal of organics in a $\mathrm{Fe}^{0} / \mathrm{H}_{2} \mathrm{O}$ process.

Therefore, in the present study, PNP was chosen as target contaminant to investigate the effect of selected factors on PNP degradation by $\mathrm{Fe}^{0}$. The objectives of this study are to (1) compare the removal rates of PNP by multiple ZVI samples with or without WMF, (2) assess the effects of $\mathrm{pH}$, initial ZVI loadings, PNP concentration, and magnetization time on PNP degradation by $\mathrm{Fe}^{0}$ with or without WMF, (3) determine the effects of several background anions on PNP degradation by $\mathrm{Fe}^{0}$ with or without WMF, and (4) clarify PNP degradation pathways by $\mathrm{Fe}^{0}$.

\section{Experimental}

\section{Materials}

PNP of 99\% purity was purchased from Sigma-Aldrich Co. LLC. (St. Louis, MO, USA). Six commercial ZVI ( $\geq 99 \%$ pure) samples including granular ZVI (about 400 mesh) and microscale ZVI were supplied by Sinopharm Chemicals Reagent Co., Ltd. (Shanghai, China), Shanghai Yunfu Nanotechnology Co., Ltd. (Shanghai, China), Shanghai Haotian Nanotechnology Co., Ltd. (Shanghai, China), and Sigma-Aldrich Co. LLC. (St. Louis, MO, USA). All chemicals were used without further purification, and solutions were prepared with deionized (DI) water.

\section{Batch experiments}

The batch experiments were conducted in a plexiglass reactor (14 cm height $\times 10 \mathrm{~cm}$ diameter) open to the air at $20 \pm 1.0^{\circ} \mathrm{C}$, and $0.5 \mathrm{~L}$ solution containing PNP was completely mixed by digital display electric blender at $500 \mathrm{rpm}$. The nonuniform WMF was generated by positioning two thin cylindrical neodymium-iron-boron permanent magnets at the bottom of the reactor. To maintain $\mathrm{pH}$ of the solutions constant $( \pm 0.1)$, buffers of $100 \mathrm{mM}$ sodium acetate, $100 \mathrm{mM}$ 2-( $N$-morpholino) ethanesulfonic acid (MES), and $40 \mathrm{mM}$ piperazine- $N, N^{\prime}$-bis(ethanesulfonic acid) (PIPES) were employed for $\mathrm{pH}$ 4.0-5.0, 6.0, and 7.0, respectively. The negligible interferences from MES and PIPES buffers forming non-complexes with $\mathrm{Fe}(\mathrm{II})$ or $\mathrm{Fe}(\mathrm{III})$ in these reactions have been extensively discussed. ${ }^{23}$ Experiments were initiated after addition of ZVI into the reactor. For the kinetic study, at fixed time intervals, a $10 \mathrm{~mL}$ sample was rapidly transferred into a $25 \mathrm{~mL}$ beaker which was immediately quenched with $100 \mu \mathrm{L}$ of methanol, filtered with $0.22 \mu \mathrm{m}$ membrane and collected into sample vials quickly.

\section{Chemical analysis}

A high-performance FE20-FiveEasy $\mathrm{pH}$ meter with a saturated $\mathrm{KCl}$ solution as electrolyte produced by Mettler-Toledo (Switzerland) was employed to measure solution $\mathrm{pH}$, and daily calibration with standard buffers ( $\mathrm{pH} 4.00,6.86$ and 9.18) was necessary to ensure its accuracy.

The BET surface areas of all ZVI samples were determined by Quantachrome Instruments Quadrasorb SI-MP-21 (Boynton Beach, FL, USA) and are listed in Table S1. $\dagger$

PNP was analysed by a Waters ACQUITY ultra-performance liquid chromatography (UPLC) system including a binary solvent manager (BSM) and a sample manager (SM) with a UV detector (TUV) (Milford, MA, USA). Separation was accomplished with an UPLC BEH C18 column $(2.1 \times 50 \mathrm{~mm}, 1.7 \mu \mathrm{m}$; Waters) at $35 \pm 1.0{ }^{\circ} \mathrm{C}$ with a mobile phase of two effluents (effluent A: $30 \%$ acetonitrile with $0.1 \%$ formic acid; effluent B: $70 \% \mathrm{H}_{2} \mathrm{O}$ with $0.1 \%$ formic acid) at a flow rate of $0.1 \mathrm{~mL} \mathrm{~min}^{-1}$. Concentrations of PNP were determined by comparing the peak area at $318 \mathrm{~nm}$ with that of standards. The intermediate products of PNP degradation were separated by the Agilent 1290 Infinity interfaced with a triple quadrupole mass detector (6400) (UHPLC-MS) (Santa Clara CA, United States). Mass spectral analysis was conducted in positive and negative mode electrospray ionization $((+)$ ESI and (-)ESI) over a mass range of 50-300 $\mathrm{m} / \mathrm{z}$. The fragment used was $110 \mathrm{~V}$ conducted in auto full scan mode (MS). Details of the UHPLC method are similar to the conditions for SMX quantification mentioned above.

\section{Results and discussion}

\section{Influence of WMF on the kinetics of PNP removal by multiple ZVI samples}

The raw time courses of aquatic PNP degradation by various ZVI samples (i.e., Guoyao, Sigma, HT1, YF1, HT2, and YF2) with and without WMF are presented in Fig. $\mathrm{S} 1, \dagger$ and all kinetic data are used in subsequent kinetic analyses. Obviously, WMF exhibited various degrees of enhancement on the reactivity of multiple ZVI samples toward PNP removal and promoted the corresponding $\mathrm{Fe}(\mathrm{II})$ release during the process of PNP removal by ZVI (as shown in Fig. S2 $\dagger$ ). A pseudo-first-order kinetic model, ${ }^{24}$ as shown in eqn (1), was employed to quantitatively describe the influence of WMF on the reactivity of ZVI. Simulative results of PNP elimination are illustrated by the solid or dashed lines in Fig. S1, $\dagger$ and the calculated rate constants of PNP removal by ZVI with and without WMF are summarized in Fig. 1.

$$
-\frac{\mathrm{d}[\mathrm{PNP}]}{\mathrm{d} t}=k_{\mathrm{obs}}[\mathrm{PNP}]=k_{\mathrm{SA}} \rho_{\mathrm{a}}[\mathrm{PNP}]
$$




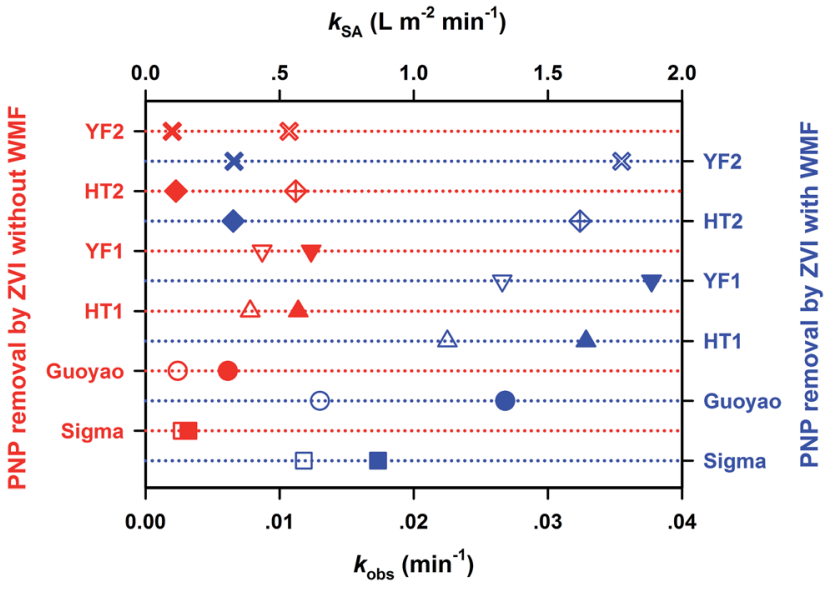

Fig. 1 Pseudo-first-order rate constants ( $k_{\mathrm{obs}}, \mathrm{min}^{-1}$, empty symbols) and normalized specific reaction rate constants $\left(k_{\mathrm{SA}}, \mathrm{L} \mathrm{m}^{-2} \mathrm{~min}^{-1}\right.$, filled symbols) of PNP removal by ZVI without (left ordinate) and with (right ordinate) WMF. Reaction conditions: $\left[\mathrm{Fe}^{0}\right]=0.10 \mathrm{~g} \mathrm{~L}^{-1}$, [PNP] $=10 \mu \mathrm{M}$, $[\mathrm{NaAc}-\mathrm{HAc}]=0.10 \mathrm{M}(\mathrm{pH} 4.0), \mathrm{rpm}=500, T=20 \pm 1^{\circ} \mathrm{C}$.

where $k_{\text {obs }}$ is the pseudo-first-order rate constant $\left(\mathrm{min}^{-1}\right)$ of PNP removal by ZVI, $k_{\mathrm{SA}}$ is the normalized specific reaction rate constant $\left(\mathrm{L} \mathrm{m}^{-2} \min ^{-1}\right)$ of PNP removal, and $\rho_{\mathrm{a}}$ is the surface area concentration of $\mathrm{ZVI}\left(\mathrm{m}^{2} \mathrm{~L}^{-1}\right.$ of solution, calculated from Table $\mathrm{S} 1 \dagger)$. The rate constants provided by Fig. 1 reveal that different $k_{\text {obs }}$ values of PNP removal by ZVI **samples vary by origins. Moreover, WMF significantly increases the rate constants of PNP removal by all applied ZVI samples. Although the increase is not uniform for different ZVI samples, the mean value of rate constants was increased appreciably from 0.0073 to $0.0236 \mathrm{~min}^{-1}$ after the WMF radiation. However, the value of $k_{\text {obs }}$ cannot really exhibit the reactivity of ZVI toward a specific contaminant, even under the same experimental conditions for ZVI, from different sources with different iron surface area concentrations. Thus, the $k_{\text {obs }}$ data should be normalized to the specific rate constants $\left(k_{\mathrm{SA}}\right)$ by eqn (1), and the obtained $k_{\mathrm{SA}}$ values are summarized in Fig. 1 . The mean value of $k_{\mathrm{SA}}$ was increased appreciably from 0.311 to $1.066 \mathrm{~L} \mathrm{~m}^{-2} \mathrm{~min}^{-1}$ after the radiation of WMF.

Meanwhile, the $\mathrm{Fe}(\mathrm{II})$ concentration increased almost linearly with time with the degradation of PNP, and a zero-order reaction kinetic model ${ }^{25}$ (eqn (2)) was employed to quantitatively describe the influence of WMF on the release of $\mathrm{Fe}(\mathrm{II})$. The simulative kinetics of $\mathrm{Fe}(\mathrm{II})$ release from $\mathrm{ZVI}$ with and without WMF at $\mathrm{pH}$ 4.0 buffered with NaAc-HAc, open to the air, are shown in Fig. S2, $\uparrow$ and the calculated Fe(II) release rate constants ( $\left.k_{\mathrm{Fe}(\mathrm{II}) \text { obs }}\right)$

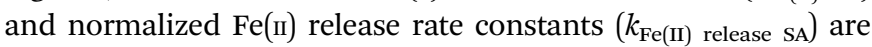
summarized in Fig. S3. $\dagger$ Obviously, WMF significantly increased the normalized $\mathrm{Fe}$ (II) release rate, which indicates that ZVI corrosion was enhanced after the addition of WMF.

$$
\frac{\mathrm{d}[\mathrm{Fe}(\mathrm{II})]}{\mathrm{d} t}=k_{\mathrm{Fe}(\mathrm{II}) \text { obs }}=k_{\mathrm{Fe}(\mathrm{II})} \text { release } \mathrm{SA} \rho_{\mathrm{a}}
$$

where $k_{\mathrm{Fe}(\mathrm{II}) \text { obs }}$ represents the zero-order rate constant $\left(\mathrm{mg} \mathrm{L}^{-1}\right.$ $\mathrm{min}^{-1}$ ) of $\mathrm{Fe}(\mathrm{II})$ release, and $k_{\mathrm{Fe}(\mathrm{II})}$ release $\mathrm{SA}$ is the normalized $\mathrm{Fe}(\mathrm{II})$ release rate constant $\left(\mathrm{mg} \mathrm{m}^{-2} \mathrm{~min}^{-1}\right)$.
Generally, it is expected that the rate constants of PNP removal by different types of ZVI under identical reaction conditions are positively related to the $\mathrm{Fe}(\mathrm{II})$ release of these ZVI samples. The investigation of Li et al. ${ }^{22}$ revealed the Pearson correlation of the specific rate constants for heavy metal removal with the specific rate constant of $\mathrm{Fe}(\mathrm{II})$ generation. Hence, the Pearson correlations of the rate constants ( $\left.\lg k_{\mathrm{SA}}\right)$ for PNP removal by six ZVI samples with the release rate constant

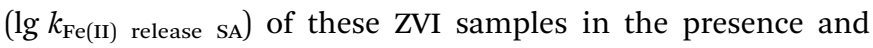
absence of WMF are demonstrated in Fig. 2.

The correlation coefficients of $\lg k_{\mathrm{SA}}$ for PNP removal by six types of ZVI with $\lg k_{\mathrm{Fe}(\mathrm{II}) \text { release SA }}$ in the presence and absence of WMF were 0.96 and 0.80 , respectively. The good correlation coefficients indicated that the degradation of PNP was accompanied by a release of $\mathrm{Fe}(\mathrm{II})$. Thus, the results of the Pearson correlations can be employed for the removal rate constant of the contaminant by a ZVI sample with WMF and without WMF from its $\mathrm{Fe}(\mathrm{II})$ release rate.

\section{Effects of treatment factors tested}

Effect of pH. The effect of WMF on PNP removal by ZVI (as shown in Fig. 3) and the variation of $\mathrm{Fe}(\mathrm{II})$ release (as shown in Fig. $\mathrm{S} 4 \dagger$ ) in this process are investigated in the $\mathrm{pH}$ range of 4.07.0. In the absence of WMF, the kinetics of PNP removal by ZVI exhibited a short lag phase of about $5 \mathrm{~min}$ before the initiation of a rapid removal period at $\mathrm{pH} 4.0$ and 5.0. The lag period of PNP removal by ZVI increased to $300 \mathrm{~min}$ as $\mathrm{pH}$ increased to 6.0. The increasing lag period with $\mathrm{pH}$ increase was ascribed to the formation of secondary reductants before the reduction of contaminants and the removal of the iron oxide layer. ${ }^{25}$ When $\mathrm{pH}$ increased to 7.0, PNP was hardly degraded by ZVI during 480 min of mixing, which indicated that the reactivity of ZVI was significantly weakened at neutral $\mathrm{pH}$. The release of Fe(II) also

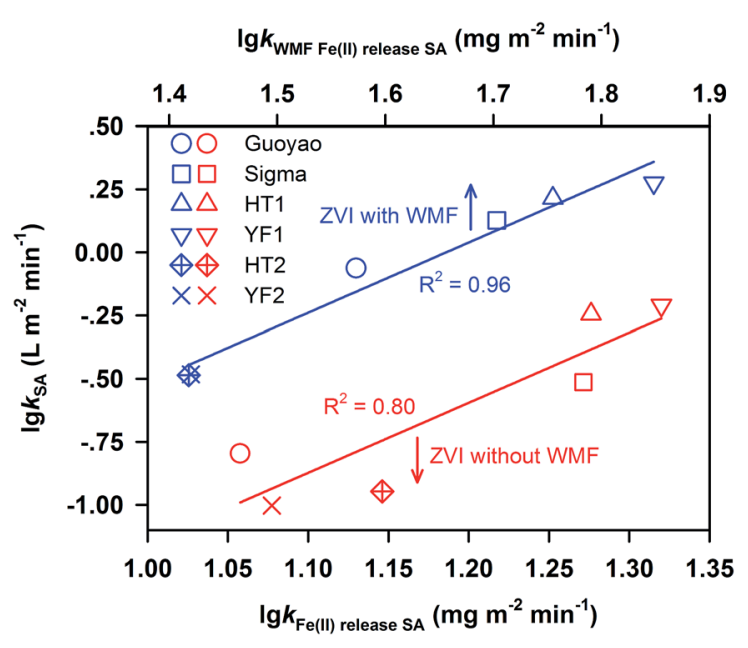

Fig. 2 Pearson correlation of the specific rate constants $\left(\lg k_{\mathrm{SA}}, \mathrm{L} \mathrm{m}^{-2}\right.$ $\mathrm{min}^{-1}$ ) of PNP removal by $\mathrm{ZVI}$ with the release rate constant ( $\lg k_{\mathrm{Fe}(I I)}$ release SA or $\lg k_{\mathrm{WMF}}$ Fe(II) release $\mathrm{SA}, \mathrm{mg} \mathrm{m}^{-2} \mathrm{~min}^{-1}$ ) of these $\mathrm{ZVI}$ samples in the presence and absence of WMF. Reaction conditions: $\left[\mathrm{Fe}^{0}\right]=0.10 \mathrm{~g} \mathrm{~L}^{-1},[\mathrm{PNP}]=10 \mu \mathrm{M},[\mathrm{NaAc}-\mathrm{HAc}]=0.10 \mathrm{M}(\mathrm{pH} 4.0), \mathrm{rpm}$ $=500, T=20 \pm 1{ }^{\circ} \mathrm{C}$. 


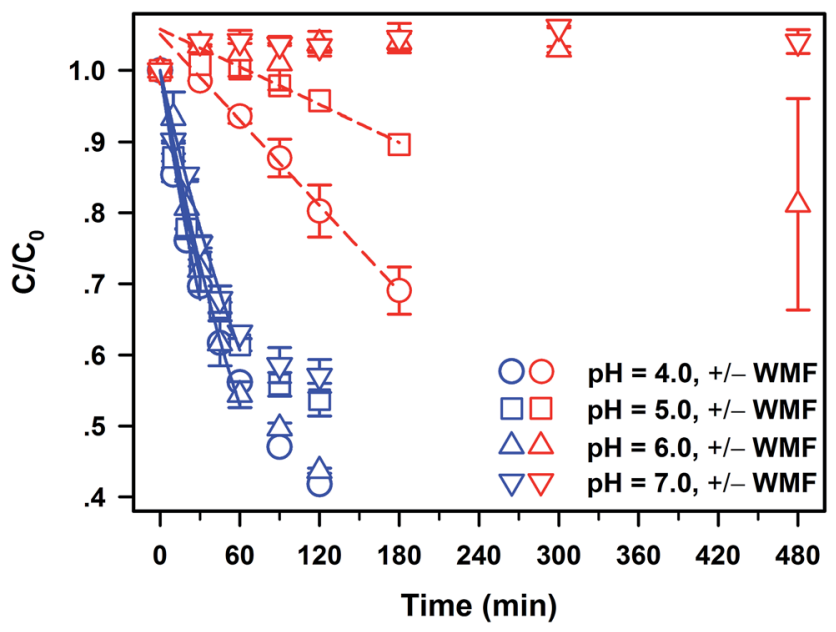

Fig. 3 Effects of $\mathrm{pH}$ on PNP removal by ZVI with and without WMF.

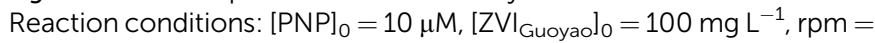
500 , and $T=20 \pm 1{ }^{\circ} \mathrm{C}$. Solid and dashed lines represent simulative results.

proved that the corrosion of ZVI was dependent on $\mathrm{pH}$. As mentioned above, the presence of WMF accelerated the removal of PNP at $\mathrm{pH}$ 4.0, which was in accordance with the release of $\mathrm{Fe}(\mathrm{II})$. The positive effect of WMF on PNP removal and $\mathrm{Fe}(\mathrm{II})$ release was also observed at $\mathrm{pH}$ 5.0. More importantly, the application of WMF significantly enhanced the removal of PNP at pH 6.0 and 7.0. The removal rate of PNP (at $120 \mathrm{~min}$ ) by ZVI reached $56 \%$ and $43 \%$ at $\mathrm{pH} 6.0$ and 7.0 , respectively. Generally, the degradation of PNP was ascribed to the reduction of $\mathrm{ZVI}$ and oxidation of ${ }^{\circ} \mathrm{OH}$ at $\mathrm{pH} 4.0$ and 5.0, and the reduction of ZVI and oxidation of the ferryl ion (Fe(IV)) generated by $\mathrm{Fe}(\mathrm{II}) / \mathrm{O}_{2}$ at $\mathrm{pH} 6.0$ and 7.0. ${ }^{13,23}$ The efficiency of the reduction by ZVI and oxidation of ${ }^{\circ} \mathrm{OH}$ or $\mathrm{Fe}(\mathrm{Iv})$ was based on the corrosion of ZVI and generation of Fe(II). As shown in Fig. S4, $\uparrow$ the application of WMF-enhanced iron corrosion of $\mathrm{ZVI}$ at $\mathrm{pH} 4.0-7.0$ and the release rate of Fe(II) showed very little difference with increasing $\mathrm{pH}$ from 4.0 to 7.0. The irregular variation of PNP removal rate by ZVI with WMF at various $\mathrm{pH}$ levels indicated that the contribution of oxidative species generated by ZVI and the reduction by ZVI was different with $\mathrm{pH}$ increasing from 4.0 to 7.0.

\section{Effect of initial ZVI loading}

The effect of initial ZVI loading from 100 to $500 \mathrm{mg} \mathrm{L}^{-1}$ on PNP removal by ZVI with and without WMF open to the air, and the variation of $\mathrm{Fe}(\mathrm{II})$ release kinetics in this process, are shown in Fig. S5. $\dagger$ In addition, simulative results of PNP elimination by eqn (1) and of $\mathrm{Fe}(\mathrm{II})$ release by eqn (2) are illustrated by the solid or dashed lines in Fig. S5. $\dagger$ In the absence of WMF, the lag phase was shortened with increased ZVI loading. Kinetics of $\mathrm{Fe}(\mathrm{II})$ release also showed a similar variation of the short lag period, which is consistent with the results of PNP removal. Thus, increasing ZVI loading can shorten the lag period and accelerate the removal of contaminants. To quantitatively describe the influence of ZVI loading on PNP removal by ZVI and Fe(II) release, linear correlations of $k_{\mathrm{obs}}$ of PNP removal and $k_{\mathrm{Fe}(\mathrm{II}) \text { obs }}$ of $\mathrm{Fe}(\mathrm{II})$ release in the presence and absence of WMF with ZVI loading are shown in Fig. 4 . The good correlation between $k_{\text {obs }}$ (or $\left.k_{\mathrm{Fe}(\mathrm{II}) \text { obs }}\right)$ and ZVI loading mainly was ascribed to the linear increase of ZVI surface area, which contributed to PNP removal and $\mathrm{Fe}(\mathrm{II})$ release. The application of WMF-enhanced PNP removal by ZVI eliminated the lag period. The enhancing factor of WMF, as the ratio of the slope in linear regression results, is stable with increasing ZVI loading, which indicates that WMF does not weaken the enhancement on ZVI reactivity with increasing ZVI loading.

\section{Effect of initial PNP concentration}

Fig. S6 $†$ illustrates the effect of initial PNP concentration $\left(C_{0}\right)$ ranging from 5 to $100 \mu \mathrm{M}$ on PNP removal by ZVI and Fe(II) release during these processes. The initial PNP concentration showed slight influence on PNP removal and Fe(II) release. The addition of WMF also accelerated PNP removal and enhanced $\mathrm{Fe}(\mathrm{II})$ release, and increasing PNP concentration inhibited the enhancing effect of WMF. As shown in Fig. 5, degradative rates $\left(k_{\mathrm{obs}} \times C_{0}\right)$ of PNP and Fe(II) release rates $\left(k_{\mathrm{Fe}(\mathrm{II}) \text { obs }} \times C_{0}\right)$ linearly increase with increasing initial PNP concentrations in the presence and absence of WMF. This behaviour was well fitted by first-order kinetics (eqn (3)) similar to the initial phase of the modified Langmuir-Hinshelwood equation, which has been applied in contaminant removal by ZVI. ${ }^{26}$ Thus, WMF does not change the kinetics of PNP removal by ZVI and Fe(II) release but improves the identical reactivity of ZVI $\left(V_{m}\right)$ towards PNP.

$$
-\frac{\mathrm{d}[\mathrm{PNP}]}{\mathrm{d} t}=\frac{V_{\mathrm{m}}[\mathrm{PNP}]}{K_{1 / 2}+[\mathrm{PNP}]}=\frac{V_{\mathrm{m}}}{K_{1 / 2}}[\mathrm{PNP}] \quad\left(\text { when } K_{1 / 2} \gg[\mathrm{PNP}]\right)
$$

where $V_{\mathrm{m}}$ represents the maximum reaction rate $\left(\mu \mathrm{M} \mathrm{min}{ }^{-1}\right)$ and $K_{1 / 2}$ is a constant reflecting the affinity of the iron surface for PNP.

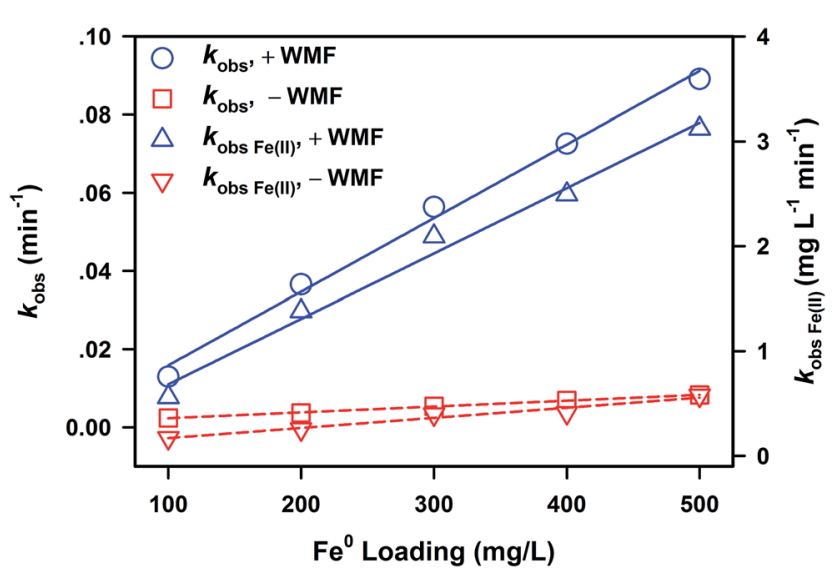

Fig. 4 Linear correlations of $k_{\text {obs }}$ of PNP removal by $Z \mathrm{VI}_{\text {Guoyao }}$ and $k_{\mathrm{Fe}(I I)}$ obs of $\mathrm{Fe}($ II) release in the presence and absence of WMF with ZVI loading. Reaction conditions: $[\mathrm{PNP}]=10 \mu \mathrm{M},[\mathrm{NaAc}-\mathrm{HAc}]=0.10 \mathrm{M}(\mathrm{pH}$ 4.0), $\mathrm{rpm}=500, T=20 \pm 1{ }^{\circ} \mathrm{C}$. Solid and dashed lines represent simulative results. 


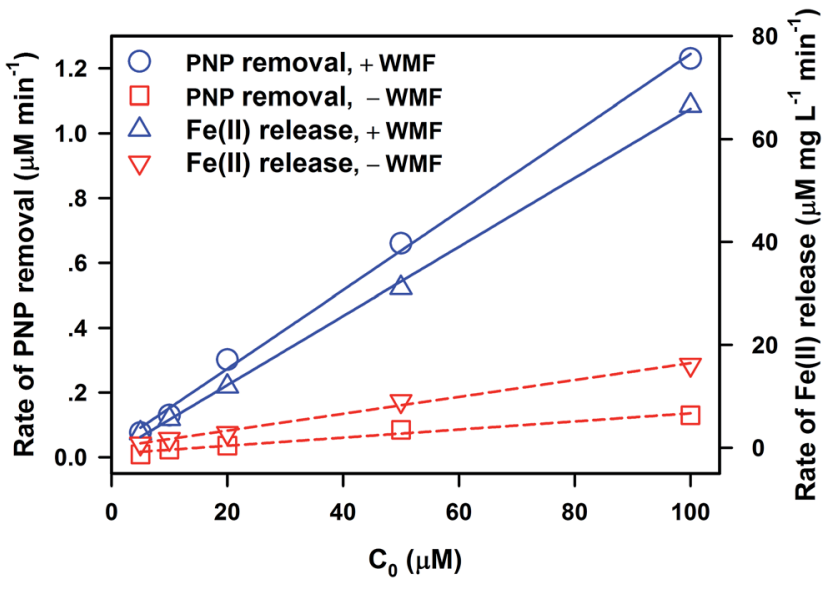

Fig. 5 Effect of initial PNP concentration on degradative rates ( $k_{\text {obs }} \times$ $C_{0}$ ) of PNP removal by $Z \mathrm{VI}$ and $\mathrm{Fe}(\mathrm{II})$ release rates ( $k_{\mathrm{Fe}(\mathrm{II}) \text { obs }} \times \mathrm{C}_{0}$ ) during these processes. Reaction conditions: $\left[\mathrm{ZVI}_{\text {Guoyao }}\right]_{0}=100 \mathrm{mg} \mathrm{L}^{-1}$ $[\mathrm{NaAc}-\mathrm{HAc}]=0.10 \mathrm{M}(\mathrm{pH} 4.0), \mathrm{rpm}=500, T=20 \pm 1{ }^{\circ} \mathrm{C}$. Solid and dashed lines represent simulative results.

\section{Effect of magnetization time}

WMF-enhanced PNP removal by ZVI, by applying a weak magnetic field in a large treatment unit during the reaction, should overcome the increasing cost of operating magnetic field devices. Hence, Guan et al. ${ }^{\mathbf{1 9 , 2 2}}$ developed ZVI premagnetization before the reaction to enhance the reactivity of ZVI toward various contaminants. However, the reactivity of premagnetized ZVI was not stable and decreased with time before the reaction. In this study, the in situ variation of ZVI reactivity towards PNP with magnetization time was studied. Fig. 6 shows the effect of magnetization time on PNP removal kinetics by ZVI at $\mathrm{pH} 4.0$, and measured reaction time courses of $\mathrm{Fe}(\mathrm{II})$ release during these processes are also summarised in Fig. S7. $\uparrow$ The enhanced reactivity of ZVI towards PNP increased with increasing magnetization time from 1 to $120 \mathrm{~min}$. The effect of

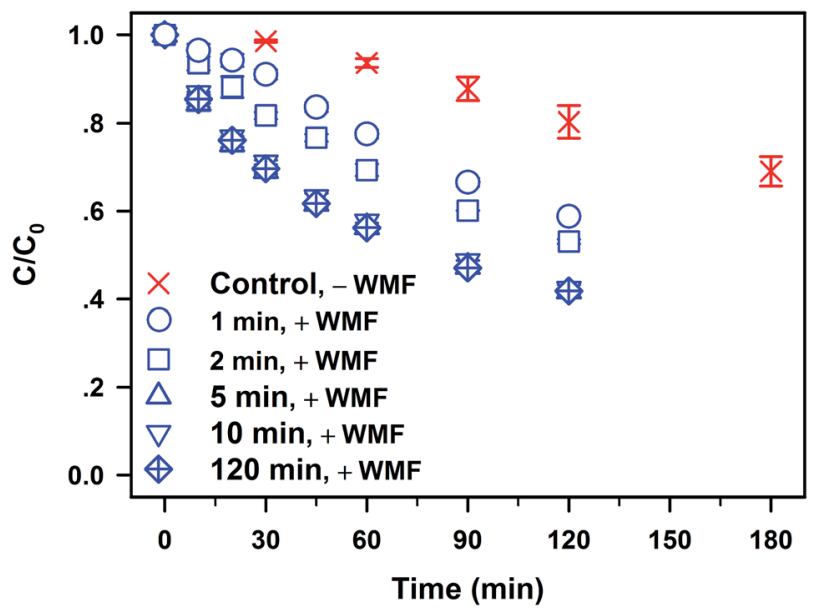

Fig. 6 Effect of magnetization time on PNP removal kinetics by ZVI. Reaction conditions: $\left[\mathrm{ZVI}_{\mathrm{Guoyao}}\right]_{0}=100 \mathrm{mg} \mathrm{L}^{-1}$, [PNP] $=10 \mu \mathrm{M}$, [NaAc$\mathrm{HAc}]=0.10 \mathrm{M}(\mathrm{pH} 4.0), \mathrm{rpm}=500, T=20 \pm 1^{\circ} \mathrm{C}$. magnetization time on PNP removal rate and $\mathrm{Fe}(\mathrm{II})$ release rate could be ignored in the presence of WMF for more than $5 \mathrm{~min}$. The high efficiency of PNP removal by ZVI with WMF in a short magnetization time was interpreted as the magnetic memory of ZVI, maintaining ZVI reactivity during the reaction processes. ${ }^{22}$ Shortened magnetization time may give engineering implications on the application of WMF on ZVI.

\section{Effects of common dissolved anions on WMF-enhanced PNP removal by ZVI}

Batch kinetics were conducted to study PNP removal by ZVI with and without WMF in the presence of various anions including $\mathrm{Cl}^{-}, \mathrm{SO}_{4}{ }^{2-}, \mathrm{NO}_{3}{ }^{-}$, and $\mathrm{ClO}_{4}{ }^{-}$. The raw time courses of PNP removal by ZVI are shown in Fig. S8. $\uparrow$ The measured reaction time courses of PNP removal during these processes are modelled by eqn (1), and corresponding rate constants are summarized in Fig. 7. Sulfate and chloride anions always exhibited positive effects on PNP removal regardless of the presence or absence of WMF. Sulfate could remove iron oxides and hydroxides from the iron surface, which increased the reactive sites of ZVI reacted with PNP. Although Sugimoto and Wang $^{27}$ reported that a high sulfate concentration beyond $50 \mathrm{mg}$ $\mathrm{L}^{-1}$ might inhibit the reactivity of ZVI in the precipitation of acicular $\alpha-\mathrm{FeOOH}$ and precipitation of basic ferric sulfate iron surface, sulfate always enhanced PNP removal, even at $50 \mathrm{mM}$, in this study. Chloride could passivate oxides on the iron surface and form strong complexes with ZVI. ${ }^{28}$ Thus, oxides on reactive sites were cleaned by chloride, and PNP removal was accelerated. However, nitrate anions always exhibited diverse effects on PNP removal in the presence and absence of WMF. Since nitrate was reduced by ZVI, reactivity of ZVI towards PNP was decreased for the competition of nitrate. ${ }^{28}$ Curiously, it was found in this study that the presence of WMF and nitrate did not decrease PNP removal rate or even exhibit a slightly positive effect on PNP removal. Perchlorate anions inhibited PNP removal by ZVI because perchlorate could occupy some

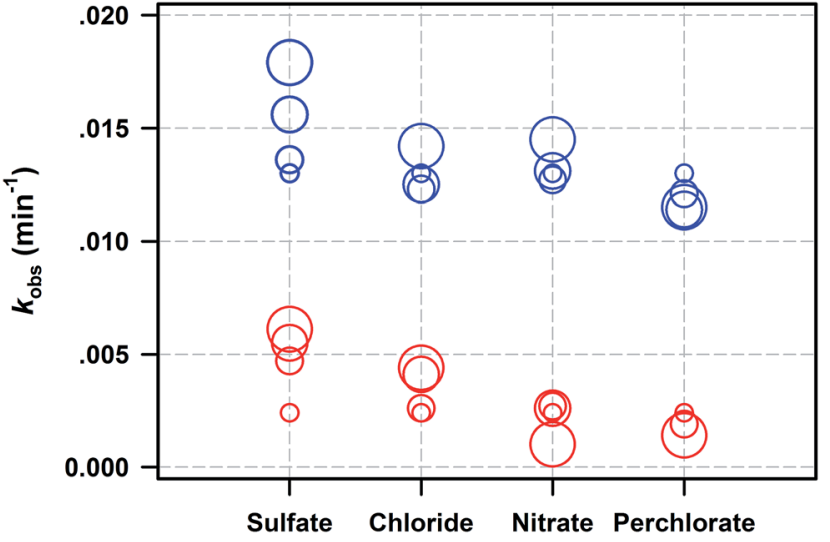

Fig. 7 Summary of $k_{\text {obs }}$ for PNP removal by ZVI vs. dissolved anions. The circle size represents the concentration of anions. The values of $k_{\text {obs }}$ for all these data are given in Table S2. $\uparrow$ Reaction conditions: $\left[\mathrm{ZVI}_{\text {Guoyao }}\right]_{0}=100 \mathrm{mg} \mathrm{L}^{-1},[\mathrm{PNP}]=10 \mu \mathrm{M},[\mathrm{NaAc}-\mathrm{HAc}]=0.10 \mathrm{M}(\mathrm{pH}$ 4.0), $r p m=500, T=20 \pm 1{ }^{\circ} \mathrm{C}$. 
reactivity sites of ZVI with PNP, which decreased PNP removal rate. In addition, WMF could not reverse the negative effect of perchlorate on PNP removal by ZVI. Overall, in the presence of various anions, the application of WMF was considered as a superior strategy for contaminant removal by ZVI.

\section{Possible degradation pathways of PNP by ZVI with and without WMF}

Many studies have identified the degradation intermediates of PNP by various processes. The species of reaction intermediates and degradation pathways of PNP removal, even in different AOPs identified by GC-MS, LC-MS, and HPLC, were different. In this study, UHPLC-MS was employed to detect the reaction intermediates of PNP by ZVI at pH 4.0, based on which the PNP degradation pathways were proposed.

It was found that WMF had no influence on the varieties of detected intermediates, which indicated that WMF enhanced PNP removal without changing the PNP degradation pathways. Five reaction intermediates were detected in the process of PNP removal, which are summarized in Table $\mathrm{S} 3 . \dagger$ Based on the detected intermediates specified in this study, two proposed transformation pathways (I and II) by ZVI leading to PNP degradation are presented in Fig. 8. In pathway I, reduction of PNP formed P1 ( $p$-nitrosophenol), and P1 was reduced to P2 ( $p$ aminophenol). This degradation pathway is considered as the classical reductive process also observed in PNP reduction by nZVI. ${ }^{3}$

It has been reported that the reaction of ZVI or nZVI and oxygen could produce reactive oxidants capable of oxidizing organics. ${ }^{29}$ Oxidation under acidic conditions is attributable to the generation of $\mathrm{H}_{2} \mathrm{O}_{2}$ during $\mathrm{ZVI}$ oxidation, which then reacts

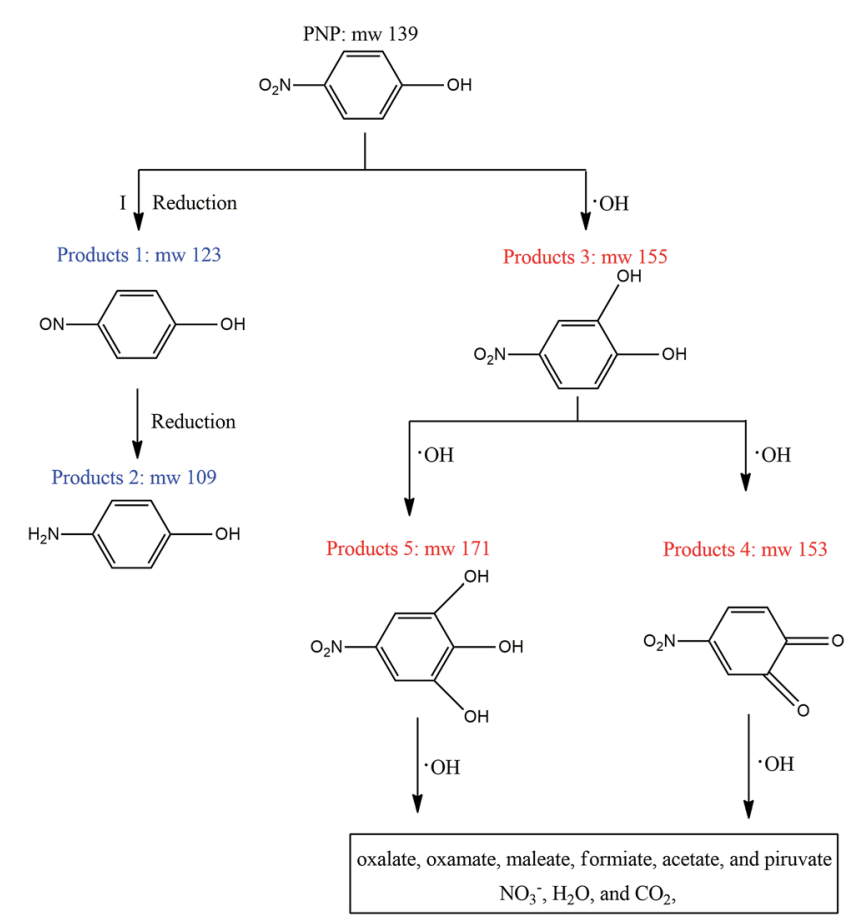

Fig. 8 Possible degradation pathways of PNP by ZVI. with $\mathrm{Fe}(\mathrm{II})$ via the Fenton reaction to produce ${ }^{\circ} \mathrm{OH}$. Thus, the second main reaction pathway (II) was PNP oxidation by ${ }^{\circ} \mathrm{OH}$. It is well known that the reaction of ${ }^{\circ} \mathrm{OH}$ with aromatic groups occurs via electrophilic addition. ${ }^{9,23}$ In pathway II, electrophilic addition on the aromatic ring by ${ }^{\circ} \mathrm{OH}$ formed P3 ( $p$-nitrocatechol), which could also be frequently detected in AOPs processes, as demonstrated in Fig. 8. On the subsequent ${ }^{\circ} \mathrm{OH}$ attack, the PNP was converted into P4 ( $p$-nitropyrogallol) or P5 ( $o$-nitrobenzoquinone). The further oxidation of P4 and P5 by 'OH resulted in the aromatic ring opening, formation of aliphatic acids, and eventual generation of mineralization products.

\section{Conclusions}

The application of WMF induced a significant enhancement in PNP removal rates by various ZVI samples from different origins at $\mathrm{pH}$ 4.0. The strong correlation between the specific reaction rate constants $\left(k_{\mathrm{SA}}\right)$ of PNP removal by various ZVI samples and the specific rate constants of $\mathrm{Fe}(\mathrm{II})$ release $\left(k_{\mathrm{Fe}(\mathrm{II}) \text { release SA }}\right)$ during these processes indicated that the reactivity of different ZVI samples depended on the release of $\mathrm{Fe}(\mathrm{II})$ and that the presence of WMF promoted iron corrosion and Fe(II) generation. WMF significantly accelerated PNP removal at $\mathrm{pH}$ 4.0-7.0, which was ascribed to the enhanced corrosion of ZVI in the presence of WMF. $k_{\mathrm{SA}}$ and $k_{\mathrm{Fe}(\mathrm{II})}$ release sA, in the presence or absence of WMF, linearly increased with increasing ZVI loading, which suggested that the enhancing factor of WMF was independent of ZVI loading. Furthermore, PNP concentration exhibited very slight effect on PNP removal with and without WMF. PNP removal rate by ZVI did not decrease after 5 min of magnetization by WMF compared with $120 \mathrm{~min}$, which was ascribed to the magnetic memory of ZVI. In addition, the short magnetization time by WMF might give an important implication on the economy and feasibility of engineering application. Effects of sulfate, chlorate, nitrate, and perchlorate anions at various concentrations on WMF-enhanced PNP removal by ZVI were investigated. WMF exhibited a positive effect on PNP removal in the presence of sulfate, chlorate, and nitrate. Although perchlorate could inhibit PNP removal by ZVI, WMF decreased the negative effect of perchlorate on PNP removal by ZVI. Furthermore, degradation pathways of PNP were proposed according to the intermediates determined by UHPLC-MS, and WMF enhanced PNP removal but did not change the PNP degradation pathways.

\section{Acknowledgements}

This work was financially supported by the National Natural Science Foundation of China (51678188) and the State Key Laboratory of Urban Water Resource and Environment (2015TS06).

\section{References}

1 J. S. Du, B. Sun, J. Zhang and X. H. Guan, Environ. Sci. Technol., 2012, 46, 8860-8867. 
2 T. C. Wang, N. Lu, J. Li and Y. Wu, Environ. Sci. Technol., 2011, 45, 9301-9307.

3 L. Tang, J. Tang, G. M. Zeng, G. D. Yang, X. Xie, Y. Y. Zhou, Y. Pang, Y. Fang, J. J. Wang and W. P. Xiong, Appl. Surf. Sci., 2015, 333, 220-228.

4 Z. I. Bhatti, H. Toda and K. Furukawa, Water Res., 2002, 36, 1135-1142.

5 L. Keith and W. Telliard, Environ. Sci. Technol., 1979, 13, 416423.

6 B. Lai, Y. H. Zhang, R. Li, Y. X. Zhou and J. L. Wang, Chem. Eng. J., 2014, 249, 143-152.

7 S. P. Sun and A. T. Lemley, J. Mol. Catal. A: Chem., 2011, 349, 71-79.

8 G. Eichenbaum, M. Johnson, D. Kirkland, P. O'Neill, S. Stellar, J. Bielawne, R. DeWire, D. Areia, S. Bryant, S. Weiner, D. Desai-Krieger, P. Guzzie-Peck, D. C. Evans and A. Tonelli, Regul. Toxicol. Pharmacol., 2009, 55, 33-42.

9 X. M. Xiong, Y. K. Sun, B. Sun, W. H. Song, J. Y. Sun, N. Y. Gao, J. L. Qiao and X. H. Guan, RSC Adv., 2015, 5, 13357-13365.

10 M. H. Entezari and T. R. Bastami, Ultrason. Sonochem., 2008, 15, 428-432.

11 B. Lai, Y. H. Zhang, Z. Y. Chen, P. Yang, Y. X. Zhou and J. L. Wang, Appl. Catal., B, 2014, 144, 816-830.

12 L. J. Matheson and P. G. Tratnyek, Environ. Sci. Technol., 1994, 28, 2045-2053.

13 X. H. Guan, Y. K. Sun, H. J. Qin, J. X. Li, I. M. Lo, D. He and H. R. Dong, Water Res., 2015, 75, 224-248.

14 R. W. Gillham and S. F. O'Hannesin, Ground Water, 1994, 32, 958-967.
15 C. H. Xu, B. L. Zhang, L. J. Zhu, S. Lin, X. P. Sun, Z. Jiang and P. G. Tratnyek, Environ. Sci. Technol., 2016, 50, 1483-1491.

16 C. B. Wang and W. X. Zhang, Environ. Sci. Technol., 1997, 31, 2154-2156.

17 R. A. Crane and T. B. Scott, J. Hazard. Mater., 2012, 211-212, 112-125.

18 L. J. Xu and J. K. Wang, J. Hazard. Mater., 2011, 186, 256-264. 19 L. P. Liang, X. H. Guan, Z. Shi, J. L. Li, Y. N. Wu and P. G. Tratnyek, Environ. Sci. Technol., 2014, 48, 6326-6334.

20 L. P. Liang, W. Sun, X. H. Guan, Y. Y. Huang, W. Y. Choi, H. L. Bao, L. N. Li and Z. Jiang, Water Res., 2014, 49, 371-380.

21 Y. K. Sun, X. H. Guan, J. M. Wang, X. G. Meng, C. H. Xu and G. M. Zhou, Environ. Sci. Technol., 2014, 48, 6850-6858.

22 J. X. Li, H. J. Qin and X. H. Guan, Environ. Sci. Technol., 2015, 49, 14401-14408.

23 C. R. Keenan and D. L. Sedlak, Environ. Sci. Technol., 2008, 42, 1262-1267.

24 T. L. Johnson, M. M. Scherer and P. G. Tratnyek, Environ. Sci. Technol., 1996, 30, 2634-2640.

25 L. P. Liang, W. J. Yang, X. H. Guan, J. L. Li, Z. J. Xu, J. Wu, Y. Y. Huang and X. Z. Zhang, Water Res., 2013, 47, 58465855.

26 S. Nam and P. G. Tratnyek, Water Res., 2000, 34, 1837-1845. 27 T. Sugimoto and Y. S. Wang, J. Colloid Interface Sci., 1998, 207, 137-149.

28 W. Z. Yin, J. H. Wu, P. Li, X. D. Wang, N. W. Zhu, P. X. Wu and B. Yang, Chem. Eng. J., 2012, 184, 198-204.

29 S. H. Joo, A. J. Feitz and T. D. Waite, Environ. Sci. Technol., 2004, 38, 2242-2247. 